\title{
Motion planning of mounting robots with intelligent control
}

\author{
Thomas Bock* ${ }^{*}$ Alexej Bulgakow ${ }^{* *}$, Dimitry Parshin ${ }^{* * *}$ \\ * Technical University Munich, Germany, thomas.bock@bri.ar.tum.de \\ ** South Russian State Technical University (NPI), Russia a.bulgakow@gmx.de \\ ${ }^{* * *}$ Rostov State Building University, Russia, pragma_plus@mail.ru
}

\begin{abstract}
: high efficiency of mounting robotized complexes is achievable by means of adaptive control algorithms that provide motion planning and forming of control actions, taking into account conditions of manipulator, object and environment. Incomplete and unclear body of data on the object and operating space does not allow to obtain the desired control using traditional computational algorithms. The constructed control algorithms allow to detour the obstacles, move in straight and curve trajectories with the specified speed, to decrease the rate of construction's movement, to perform orientation of construction and provide its smooth installation into the projective position.
\end{abstract}

KEYWORDS: robotization, construction, control algorithms, visual sensor, mobile robots, planning of the robot's movement, kinematics.

\section{INTRODUCTION}

Successful problem solving of constructingmounting operations robotization connected with orientation and installation of elements and constructions requires application of original kinematics structures combined with adaptive and intelligent control. High efficiency of mounting robotized complexes is achievable only by means of adaptive control algorithms that provide motion planning and forming of control actions, taking into account conditions of manipulator, object and environment. Control algorithms are to take into consideration many disturbing factors: orientation errors of the elements, wind load actions, limited fabrication accuracy of constructions, non-determinancy of environment, deformation of manipulation system. In the process of construction's installation and orientation additional constraints can occur and change the plan of mounting operations. Solving of the given problems completely depends on the applied mathematical models reflecting specific character of the complex system functioning conditions. Incomplete and unclear body of data on the object and operating space does not allow to obtain the desired control using traditional computational algorithms. As a result, for control of mounting robots a problem of model construction of the mounting system arises. The model reflects real condition of mounting equipment and technoenvironment.

\section{CONTROL ALGORITHMS OF ROBOTS}

In terms of this problem solving let us consider the questions connected with receipt of data on the environment, their processing aimed at identification of the object's position and planning of its movement, to plan movement taking into account the running state of the environment. The prospective trend of robotic mounting complexes sensitization is application of visual sensors, developed on the basis of laser scanning systems, ultrasonic scanners or TV cameras. Visual sensors are able to provide robotic complexes with data on the operating area condition required for control and dynamically track possible changes in the real time mode. In this connection the problem of effective processing of visual information occurs to be urgent. A model of operating environment is to include datameasuring structures, controlling the object's position and orientation, presence of obstacles on the path of its motion. Data-base of the model is to be renewed at every stage of control before movements planning.

The most informative is a visual sensor, representing a TV camera, connected to a computer. The output information of such a sensor is displayed in the form of brightness points matrix of the sensor's field of vision and 
is described by indistinct set $S_{0}$ corresponding to the unclear condition.

$S_{0}=\left\{\left\langle\mu_{S_{0}}(i, j) /(i, j)\right\rangle\right\} \rightarrow i, j \in I$,

where $\mu_{S_{0}}(i, j)$ is the truth degree of the fact that a point with coordinates $(i, j)$ of the sensor's field of vision belongs to the obstacle or is not included into the operating area; $I=\{1,2, \ldots, m\}$ is a set of discrete coordinates, the upper level $m$ of which is determined by parameters of a technical vision system. In case the obstacles are viewed as dim-outs in the light background of the environment, the truth degree $\mu_{S_{0}}(i, j)$ is defined by a dim-out quantity of the point. In the simplest case $S_{0}$ can be considered as a square matrix of truth degrees

$S_{0}=\left\|\mu_{i, j}^{0}\right\|_{m} \rightarrow \mu_{i, j}^{0}=\mu_{S_{0}}(i, j) \rightarrow i, j \in I$.

In that case the vague term "dim-out" ("brightness") shows presence or absence of the obstacle in the operating area.

Recognition of the operating area condition is carried out on the basis of comparison of the input condition with a certain set of standard conditions, stored in a specialized database. Standard conditions are selected so that every of them could cover a certain number of possible input conditions, being an indistinct union of these conditions. As a proximity measure of the conditions a degree of indistinct equality is used. In order to decrease the summable time of this problem solving, hierarchical organization of a set of standard conditions is selected. For this reason a check of proximity measure of the conditions is performed. A set of standard conditions is divided into $L$ levels, their number depending on necessity degree of presenting details in the operating area. A condition of every level is specification of the standard conditions of the previous level and is indistinctly included into these conditions. Recognition of the obstacle's coordinates is performed by means of indistinct algorithm $A$, plotting (constructing, setting up) a correspondence between standard conditions and indistinct coordinates of the obstacle area [1]. Indistinct algorithm $A$ is specified by hierarchically organized set of conditional indistinct operators: if $\left(S_{0} \subseteq S_{1}\right)$, then $\left(x=\widetilde{X}_{1}, y=\widetilde{Y}_{1}\right)$,

if $\left(S_{0} \subseteq S_{l}\right)$, then $\left(x=\widetilde{X}_{l}, y=\widetilde{Y}_{l}\right)$,

if $\left(S_{0} \subseteq S_{n}\right)$, then $\left(x=\tilde{X}_{n}, y=\tilde{Y}_{n}\right)$,

where $S=\left\{S_{1}, S_{2}, \ldots, S_{n}\right\}$ is a set of standard blurred conditions; $(x, y)$ are the obstacle's coordinates, $\tilde{X}_{l}, \widetilde{Y}_{l}(1 \leq l \leq n)$ are indistinct obstacle's coordinates on orthogonal axes. Indistinct coordinates $\tilde{X}_{l}, \widetilde{Y}_{l}$ represent indistinct sets in a distinct (clear) set $I=\{1,2, \ldots, m\}$ :

$$
\tilde{X}_{l}=\left\{\left\langle\mu_{X_{l}}(i) / i\right\rangle\right\}, \tilde{Y}_{l}=\left\{\left\langle\mu_{Y_{l}}(i) / i\right\rangle\right\}, i \in I \text {. }
$$

The obstacles are prescribed by the expert database, and standard conditions from set $S$ are formed automatically. Standard conditions for indistinct coordinates $\widetilde{X}_{l}, \widetilde{Y}_{l}$ are plotted so that it was possible to cover all possible configurations of graphic primitives of the obstacles. Standard blurred condition $S_{i}$ is physically a certain three-dimensional area, formed by joint rotation of attribute functions $\mu_{X_{l}}$ and $\mu_{Y_{l}}$ plots around the axis, going perpendicular to the field of view plane of a visual sensor (TV camera) through point $\left(i_{X_{l}}, i_{Y_{l}}\right), \quad$ where $\quad i_{X_{l}}=\arg \max _{i \in I} \mu_{X_{l}}(i)$, $i_{Y_{l}}=\arg \max _{i \in I} \mu_{Y_{l}}(i)$.

Working out of control over the constructingmounting robot is carried out as a process of decision-making in a non-determined environment. Planning of motion is performed on the basis of discrete sequences of trajectory representation. On the basis of information about environment and the system's own condition the aim is synthesized. On its basis control is worked out and implemented. An important property of the adaptive mounting robot is its ability to independently plan movement towards the indicated purpose position in conditions of unforeseen changes in the operating environment. Realization of such control is convenient to be performed on the basis of neuronetwork models application and considering of displacement of the mounted parts (details) in the configuration space with time dimension. The obligatory condition for 
the plotted neuronetwork functioning is presence of a priori information on the environment status. Mounting works being performed in a dynamic environment, the problem of control is getting more complicated, for occasional obstacles are likely to occur while implementing trajectory movements that are to be on the neuronetwork model.

For such problems solving use the idea of a configuration space [2]. In configuration space $C \subset R^{n}$ initial and purpose configuration of the mounting robot $K_{b}, K_{e}$ are given. If at the examined moment $t_{k}$ there is $q_{k}$ of the obstacles in space $C$, they are described as a field of forbidden configurations $B_{k}$. This area is represented by a set of forbidden zones, every of them is described by a number of vectors:

$$
B_{k}=\left\{B_{i k}\right\}=\left\{\left[x_{11}, \ldots, x_{1 n}\right]^{(k)}, \ldots,\left[x_{q 1}, \ldots, x_{q n}\right]^{(k)}\right\}
$$

Position of the object of manipulation in the process of movement we describe by vector $M(t)=\left[m_{1}(t), \ldots, m_{n}(t)\right]$, bound points of movement trajectory corresponding to equalities $M\left(t_{b}\right)=K_{b}, M\left(t_{e}\right)=K_{e}$. Planning of manipulator's motion from initial to final point of the trajectory is implemented in accordance with condition $M\left(t_{k}\right) \cap B_{k}=0 \rightarrow t_{b} \leq t \leq t_{e}$. For solving the problem of planning in this case it is appropriate to use neuronetwork models; in the network dynamics they have a built-in mechanism of permanent excitation; a set of regulations for identification and indication of the robot's next position is also prescribed [3]. At every step of control such a neuronetwork model allows to introduce updating into representation of the operating environment, taking into account occurrence and travel of obstacles in the operating area. At every step of control a new wave of activity with renewed information about environment is expected to come. The applied neuronetwork model represents a multi-layered, locally connected, parallel neuron architecture. The field of the examined network consists of a set of overlapped proximity, position of neurons is defined by $n$-measured vector of configuration space $C$. Surrounding of every neuron comprises $2 n$ of adjoining neurons, directly linked to it. Such neuronetwork model is described by a set of vectors of neurons' condition $X_{i}=\left[x_{i}, \widetilde{W}_{s i}\right] \subset \mathfrak{R}^{2 n+1}$, where $x_{i}$ the level of neuron activation; $\widetilde{W}_{i}=\left[w_{i_{1} i}, \ldots, w_{i_{n} i}, \hat{w}_{i_{1} i}, \ldots, \hat{w}_{i_{n} i}\right]-$ vector of weight factor, determining the force of synapses between neurons; $w_{j i}, \hat{w}_{j i}$ - weight factors, corresponding to two different regulations of training. At the initial moment of time activity of all neurons and values of weight factors equal 0 (zero). According to [4] dynamics of such a neuronetwork model is described by a system of equations like:

$$
\begin{aligned}
& x_{i}(t+1)=\zeta_{i}+\left(1-\zeta_{i}\right) \\
& \left(\sum_{j \in s_{i}} \zeta_{j}\left(1+x_{i}(t)\right)+r(\delta) \cdot\left(1-\sum_{j=s_{i}} \zeta_{j}\right)\right),
\end{aligned}
$$

where $\zeta_{j}$ is an exciting outer input, associated with every neuron; $r(\delta)$ - function, equal to $(\delta$ )$=\bmod \left(\sum_{j=s_{i}} w_{j i}\left(x_{j}(t)+2\right)\right)$, if $\delta>0$, otherwise $r(\delta)=0$.

Planning of the robot's movement path is carried out on the basis of the formulated regulations of weight factors change

$$
\begin{aligned}
& w_{j i}(t+1)=\left\{\begin{array}{l}
1 \rightarrow P_{k}\left(X_{s_{i}}(t)\right)>0 \\
0 \rightarrow P_{k}\left(X_{s_{i}}(t)\right)<0
\end{array},\right. \\
& \hat{w}_{j i}(t+1)=x_{j}(t), j \in s_{i} .
\end{aligned}
$$

The running condition of environment is reflected by means of information entering the neurons' outer inputs. If there are any obstacles in configuration space $C$, information $o_{j}=1$ enters the outer inhibiting inputs of neurons, corresponding to these obstacles. Activity of the modeling network is initiated by a neuron, the excited input of which signal $e_{j}=1$ enters. Function of priority $P_{k}\left(X_{s_{i}}(t)\right)$ provides selection of a neuron by means of successive scans of the adjoining neurons. Neuron with $w_{k i}>0$ is selected as an active one. By that time its level of activity has changed, it is not associated with the obstacle and its level of activity does not exceed the one of $i$-neuron. The path of robot's movement is defined by motion regulation, which can be 
represented

$\tau\left(t_{s}+n\right)=\left\{p_{j}: w_{j i}+e_{j}>0, j \in s_{i}\right\}$. Vector $p_{j}$ describes robot's configuration in space $C$, associated by $j$-neuron, and train of neurons corresponds to the safe movement path.

\section{STATEMENT}

The constructed control algorithms allow to detour the obstacles, move in straight and curve trajectories with the specified speed, to decrease the rate of construction's movement, to perform orientation of construction and provide its smooth installation into the projective position.

\section{REFERENCES}

1. Melikhov A.N., Berstein L.S., Korovin S.Ya. Blurred Condition Models of Decision-Making. - Taganrog: TRTI, 1996.

2. Lozano-Perez T. Spacial planning: a configuration space approach // IEEE Transactions on Computers, 1983, pp.C32:108-120.

3. Lebedev D.V. Application of Wave Propagation Neuron Network for Planning of Safe Paths of Plane Robots // Information Technologies, № 10, 2000, pp.19-26.

4. Lebedev D.V., Steil Y.Ya. Neuronetwork Model for Paths Planning of Autonomous Robot, taking into account Dynamic Changes in the environment // Intelligent Systems, № 6, 2002, pp.195-214. 